Estudios de

lingüística inglesa aplicada

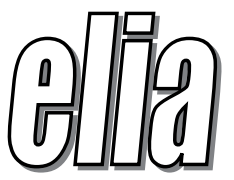

\title{
LA TRADUCCIÓN EN EL AULA DE LENGUAS PARA FINES ESPECÍFICOS: ANTIGUAS HERRAMIENTAS EN NUEVOS CONTEXTOS
}

\section{TRANSLATION IN THE CLASS OF LANGUAGES FOR SPECIFIC PURPOSES: OLD TOOLS IN NEW LEARNING CONTEXTS}

\section{Inmaculada Barbasán Ortuño}

Universitat Politècnica de València, Spain

inbaror@upvnet.upv.es

\section{Carmen Pérez-Sabater}

Universitat Politècnica de València, Spain

cperezs@idm.upv.es

\section{Begoña Montero-Fleta}

Universitat Politècnica de València, Spain

bmontero@idm.upv.es

DOI: http://dx.doi.org/10.12795/elia.2018.i18.03

La traducción ha experimentado un proceso de ostracismo durante muchos años debido a nuevas metodologías que tendian a evitar el uso de la lengua materna en el aula, al menos en estadios no iniciales de aprendizaje. Este trabajo plantea la hipótesis de si el uso pedagógico de la traducción L1-L2 en un curso de Lenguas para Fines Especificos (LFE) puede tener un papel relevante en el aprendizaje de la L2. Se analiza en detalle en qué medida las actividades de traducción de frases cortas debidamente diseñadas y 
dirigidas por el docente son una herramienta válida para mejorar la expresión escrita de dos grupos de alumnos con nivel intermedio alto de conocimientos de lengua: estudiantes anglófonos que cursan español como segunda lengua y estudiantes españoles de inglés, todos ellos cursan grados en tecnologías de la información. Las frases cortas diseñadas para practicar ciertas estructuras gramaticales como el uso del artículo de referencia genérica y vocabulario especifico de tecnologías de la información resaltan las diferencias entre los dos sistemas lingüisticos. La aplicación de una prueba de expresión escrita inicial y otra final así como un cuestionario inicial y otro final sirven para comprobar que la traducción ayuda en la mejora en el dominio de la producción escrita y que el alumnado de lenguas de especialidad actual valora mucho las tareas de traducción L1-L2 en el aula. La comparación de los dos grupos resalta las interferencias sintácticas y léxicas experimentadas en la traducción en función de la L1 del alumno.

Palabras clave: traducción pedagógica; enseñanza L2; competencia lingüística; competencia comunicativa; nivel de satisfacción del alumno.

Translation has been excluded from the language classroom for many years due to the fact that new methodologies helped avoid the use of the mother tongue in the classroom at levels other than the initial stages of learning. This study is based on the assumption that the pedagogical use of translation L1-L2 in an LSP (Language for Specific Purposes) course can have a relevant role in $L 2$ learning. The analysis examines to what extent translation activities consisting of short sentences designed by the teacher can be a valid tool to improve the writing skills of two LSP groups of upperintermediate level students: English native speakers who study Spanish as a second language and Spanish learners of English. These short sentences are aimed at learning the use of the article for generic reference and technical vocabulary in the field of computer science, which generally poses problems for this type of learners. An initial writing test and a final one together with an initial and final questionnaire designed accordingly show that translation can be a perfect tool to improve writing skills and that current LSP students value these tasks very positively. The comparison of the two groups of learners highlights the syntactic and lexical interferences each group experiences depending on their L1.

Keywords: pedagogical translation; L2 teaching; language competence; communicative competence; level of student satisfaction. 


\section{Introducción}

La enseñanza de lenguas ha estado vinculada durante mucho tiempo a la traducción; pero ya desde el siglo XIX se ha venido cuestionando su validez como método pedagógico, fundamentalmente por el hecho de que el ejercicio de traducción implica el aprendizaje del idioma a través de los patrones de la lengua materna. Sin embargo, muchos de los detractores de la traducción lo que verdaderamente rechazan es el método específico de gramática y traducción como base central en el aprendizaje de la lengua (Caballero Rodríguez, 2010; Carreres, 2006; Malmkjaer, 1998; Popovic, 2001).

Para apreciar el valor pedagógico de la traducción debemos distinguirla de la profesional. La traducción profesional suele ser traducción directa (de L2 a L1), la llevan a cabo profesionales específicamente formados para ello y se traducen textos completos más o menos largos. En cambio, la traducción pedagógica suele recurrir a la traducción de L1 a L2 (a menudo llamada inversa por los que se oponen a ella, como indica Zanettin, 2009), los aprendientes no son profesionales de la traducción y normalmente se trabaja con frases o fragmentos, y en niveles altos con textos completos (Alcarazo López y López Fernández, 2014). Asimismo, si tenemos en cuenta el fin último de la traducción, la pedagógica se utiliza como instrumento para concienciar al alumno de las diferencias semánticas y sintácticas entre las dos lenguas, a diferencia de la traducción profesional en donde la traducción del texto es el principal objetivo de la actividad, ocupando los problemas lingüísticos un segundo plano (Gile, 1995; Vermes, 2010). Para estos autores, la traducción en etapas de aprendizaje de una lengua extranjera implica la producción de textos a partir de la L1 para concienciar al alumno de las diferencias entre la lengua materna y la lengua meta.

La necesidad de profundizar en la traducción como recurso pedagógico en la enseñanza y aprendizaje de lenguas extranjeras ha sido señalada en varias investigaciones (véase, por ejemplo, Enríquez Aranda, 2003). En respuesta a esta demanda, este artículo aborda un estudio científico basado en la introducción de la traducción pedagógica en el aula de lenguas extranjeras, en un contexto de lenguas de especialidad, Lenguas para Fines Específicos (LFE), su objetivo es mejorar la producción escrita en la L2, uno de los requisitos del Marco Común Europeo de Referencia 
para las Lenguas (MCER) para el nivel B2. Al respecto, la traducción se compara en dos grupos de perfiles similares en cuanto a edad y disciplina de estudio, pero diferentes en lo que respecta a su lengua materna: el español y el inglés son las dos lenguas del estudio pero, según el grupo, estas lenguas son su L1 o su L2; es decir, hablantes nativos de inglés, en su mayoría estadounidenses, que aprenden español y hablantes de español que estudian inglés.

Este artículo está estructurado como sigue: tras una introducción al tema de estudio, detallaremos las características de la investigación realizada, los parámetros examinados y los resultados que se han obtenido. Finalmente expondremos las conclusiones y sugeriremos estudios futuros relacionados con lo tratado aquí.

\section{Marco teórico}

\subsection{La traducción en la enseñanza de lenguas}

Tradicionalmente, como se ha destacado en la introducción, las críticas al uso de la traducción en el aula no tienen que ver con la traducción como práctica sino que están relacionas con el método de enseñanza gramática y traducción, limitado básicamente al género literario (Caballero Rodríguez, 2010). Según esta misma autora, las razones de su exclusión en la enseñanza de lenguas van desde considerar que el alumno se puede frustrar al no conseguir realizar buenas traducciones hasta lo perjudicial que puede ser tener que recurrir a los patrones de la lengua materna para aprender una nueva lengua. Por el contrario, Duff (1989) fue pionero en la defensa de la traducción como una actividad plenamente válida para practicar y perfeccionar la lengua extranjera. Enríquez Aranda (2003) matiza la opinión de Duff al señalar la utilidad de la traducción en ciertas etapas del aprendizaje de una lengua extranjera y/o para ciertos fines como evaluar progresos (Catford, 1965 en Enríquez Aranda, 2003). Es más, autores como Alcarazo López y López Fernández (2014) defienden la oportunidad que supone la traducción pedagógica para explorar, más allá del significado literal de las palabras, aspectos contextuales, socioculturales y comunicativos de la lengua.

En esta línea, durante los últimos años del siglo pasado se ha reevaluado el valor positivo pedagógico de la traducción, lo que Carreres 
et al. (2017) llaman 'giro traductológico' en la enseñanza de lenguas. Sin embargo, el empleo de la traducción como recurso pedagógico todavía no está plenamente incorporado a la enseñanza porque cada vez se da más importancia a la comunicación oral frente a la escrita como destreza principal que se quiere perfeccionar en el aula de idiomas.

En el contexto europeo debemos tener en cuenta las consideraciones que puedan hacer al respecto los documentos de referencia en la enseñanza de L2 en los marcos europeo y estatal: el Marco Común Europeo de Referencia para las Lenguas (MCER) y el Plan Curricular del Instituto Cervantes. El MCER menciona la traducción principalmente en dos de sus vertientes. Por un lado, en el capítulo 4, "El uso de la lengua y el usuario o alumno" se señala la traducción como herramienta de mediación entre hablantes, al mismo nivel que la interpretación; de hecho, se propone la traducción exacta o la literaria como actividad de mediación escrita. Por otro lado, en el capítulo 6, "El aprendizaje y la enseñanza de la lengua" se recogen, someramente y sin decantarse por ninguna, algunas opciones metodológicas para el aprendizaje y la enseñanza de L2. En este punto se especifica el papel que juegan los textos en este proceso y se lanza una pregunta, ¿cómo se espera o se exige que los alumnos aprendan de textos hablados o escritos? Y una de las posibles respuestas que se ofrecen es la traducción sistemática del texto a L1 realizada por el alumno (Consejo de Europa, 2002:144).

En cuanto al Plan Curricular, en la edición de 2006 el último capítulo está dedicado a los procedimientos de aprendizaje y es dentro de estos donde se menciona brevemente la traducción, en el subapartado "Procesamiento y asimilación del sistema de la lengua", como herramienta útil para elaborar e integrar la información. En primer lugar, se define como la conversión de ideas formuladas en una lengua a otra que se conozca y, en segundo lugar, se añaden algunos ejemplos de su aplicación:

- Traducir literalmente expresiones o locuciones a la lengua materna u otras lenguas para intentar comprender su significado.

- Traducir enunciados de la lengua materna u otras lenguas al español con el fin de producir significados.

- Traducir oraciones a la lengua materna u otras lenguas con el fin de comprender y recordar las reglas gramaticales (Instituto Cervantes, B1B2, 2006: 639). 
Si bien no hay ninguna referencia explícita a la traducción pedagógica, observamos que la traducción es valorada positivamente como recurso para producir significados y para recordar y comprender la gramática.

En este trabajo seguimos a Carreres et al. (2017:104) para quienes la traducción inversa, es decir, de la lengua materna a la lengua de estudio, es "un componente particularmente fructífero en la adquisición de una lengua". Además, como apunta Zanettin (2009), es un buen recurso pedagógico para mejorar la lectura y la redacción de textos en L2 en contextos de especialidad.

\subsection{Transferencia lingüística}

El concepto de transferencia lingüística o la influencia de la L1 en la L2 se desarrolla en el marco de la teoría del análisis contrastivo aplicada a la enseñanza de lenguas extranjeras. Lado (1957) postula la existencia de dos tipos de transferencia: la positiva, que se produce cuando las estructuras que se comparan en la lengua materna y la L2 se parecen entre sí, y la transferencia negativa o interferencia, que se produce cuando las estructuras que se comparan son diferentes en ambas lenguas y desembocan en el error.

La valoración del concepto de transferencia ha evolucionado con el tiempo: ha pasado de mecanismo principal en el análisis contrastivo, a ser minimizado posteriormente en el análisis de errores para finalmente redimensionarse en la actualidad bajo un nuevo prisma en el que el aprendiente es "un participante activo en el proceso de aprendizaje y el fenómeno de la transferencia se concibe como una de las estrategias centrales que éste pone en marcha" (Alexopoulou, 2010).

De hecho, Ellis (1994) afirma que ninguna teoría de aprendizaje puede descartar la acción de la transferencia de formas de la L1 a la L2 y subraya que, además de su vertiente pasiva, hay que considerar también las activas o estratégicas, es decir, la posibilidad de que el estudiante utilice la L1 como fuente de hipótesis sobre la L2, con finalidad tanto comunicativa como de aprendizaje. En esta línea también se posiciona el Plan Curricular del Instituto Cervantes, que considera la transferencia positivamente como

ELIA 18, 2018, pp. 51-79～DOI: http://dx.doi.org/10.12795/elia.2018.118.03 
un mecanismo que supone "movilizar conocimientos de otras lenguas [...] para la comprensión o producción con éxito en la nueva lengua" (Instituto Cervantes, B1-B2, 2006:639); es decir, no la plantea como un obstáculo sino como una herramienta de aprendizaje.

Nos referiremos a continuación a los distintos tipos de interferencias L1 a L2 en el aprendizaje de un lenguaje de especialidad en las que se va a centrar este trabajo:

2.2.1 Interferencias sintácticas,

2.2.2 Interferencias en el léxico de especialidad.

\subsubsection{Interferencias sintácticas: uso del artículo en español e inglés}

Las interferencias sintácticas responden generalmente a diferencias entre la L1-L2 como, por ejemplo:

a. Diferencias en el orden de las palabras, ya que la estructura sintáctica en el caso del español es mucho más flexible que la del inglés.

b. Diferencias en la construcción de las oraciones: omisión de sujeto, el uso del artículo para referencia genérica, etc.

De entre todas ellas y ante la dificultad de tratar cada una de dichas interferencias sintácticas, en este artículo nos centraremos únicamente en el uso del artículo de referencia genérica.

En lo que respecta al uso del artículo en inglés, el concepto de referencia genérica es crucial (Quirk y Greenbaum, 2002). La omisión del artículo en plurales y nombres no contables cuando se hace referencia genérica no se corresponde con las reglas del español, que necesita del artículo determinado en estos casos, y, por tanto, suele dar problemas a los aprendientes españoles. Esto es debido básicamente a que, en general, como apuntan Snape et al. (2013), el uso del artículo para expresar referencia genérica en la L1 condiciona a los aprendientes en su elección del artículo en la L2 de forma más acusada que en otras interferencias sintácticas. 


\subsubsection{Interferencias en el léxico de especialidad}

El léxico propio es uno de los rasgos fundamentales en el aprendizaje de las lenguas de especialidad o Lenguas para Fines Específicos, ya que posee una especificidad propia de su ámbito que la distingue de la lengua común (Pérez-Sabater y Montero-Fleta, 2015). El léxico técnico debe ser foco de atención en el aula de LFE pues su presencia es patente al ocupar entre el $5 \%$ y el $21 \%$ del texto (Strevens, 1977). Muy importante también es el léxico subtécnico, que conforma el $70 \%$ del texto (Strevens, 1977), compuesto por palabras de uso frecuente en textos específicos, algunas de las cuales cambian su significado habitual al ser utilizadas en un contexto especializado. Como señalan Pérez-Sabater y Montero-Fleta (2015), en el debate existente entre los académicos sobre la conveniencia de enseñar vocabulario subtécnico común a todas las disciplinas o enseñar una lista más reducida de vocabulario técnico restringido a una disciplina, el papel de los alumnos tiene mucha importancia, ya que su dominio de la lengua de especialidad puede condicionar su trabajo futuro. En nuestro caso, hemos optado por un método ecléctico que combine los dos enfoques, vocabulario subtécnico general y vocabulario de la especialidad de tecnologías de la información.

Las principales interferencias léxicas L1-L2 se producen por:

a. Utilización de vocablos inexistentes en L2 pero con forma semejante a la Ll.

b. Utilización de vocablos existentes en L2 semejantes en forma a la L1 pero con distinto significado en ambas lenguas. Se trata de vocablos, denominados falsos amigos (false friends) o falsos cognados (false cognates), normalmente de origen latino.

c. Interferencia en la ortografía de palabras que son muy similares en L1 y L2.

d. Errores en el género de las palabras en español. Los estudiantes anglófonos presentan la dificultad añadida, con respecto a los hispanófonos, de no poseer en su lengua materna la marca de género en la mayoría de los sustantivos. La carencia de este rasgo morfológico, que es inherente a los sustantivos españoles, priva a

ELIA 18, 2018, pp. 51-79～DOI: http://dx.doi.org/10.12795/elia.2018.118.03 
estos aprendientes de la intuición innata que sí poseen otros estudiantes de lenguas maternas románicas. De este modo, en ocasiones parecen quedar a merced, incluso en el nivel de lengua que nos ocupa, de algunas reglas básicas aprendidas.

En lo que respecta al papel de la traducción en el aprendizaje del léxico técnico, Zanettin (2009) señala que la traducción es de interés para la adquisición y práctica del vocabulario de especialidad a la hora de estudiar falsos amigos, cognados, colocaciones y frases hechas. En esta línea planteamos las hipótesis de este estudio que especificamos a continuación.

\section{Hipótesis} hipótesis:

Esta investigación tiene como objetivo corroborar las siguientes

1. El uso pedagógico de la traducción puede tener un papel importante en la mejora del dominio del uso del artículo de referencia genérica en el aula de LFE y en el dominio de vocabulario de especialidad.

2. Aunque las lenguas maternas de los dos grupos contrastados son diferentes, sus respectivas interferencias lingüísticas en la producción escrita son similares.

3. La práctica de la traducción en un curso de LFE puede contribuir a la mejora de la expresión escrita.

4. La práctica de la traducción redunda en una mayor satisfacción del alumno en su aprendizaje.

\section{Metodología}

El estudio se llevó a cabo en el curso 2016-2017 con alumnos de español L2 e inglés L2 para las tecnologías de la información, asignaturas que tienen como objetivo conseguir el nivel B2 de cada lengua según el MCER. El material tratado en el curso se compone de diez temas centrados en un

ELIA 18, 2018, pp. 51-79～DOI: http://dx.doi.org/10.12795/elia.2018.118.03 
contexto técnico general y cinco temas en un contexto de especialidad. Intervinieron en el estudio dos grupos de alumnos con perfiles diferentes:

Grupo 1: 60 españoles que cursan inglés como segunda lengua en un curso de lengua extranjera para fines específicos dentro de una titulación de grado en ingeniería.

Grupo 2: 25 hablantes nativos de inglés que estudian español como segunda lengua también en un contexto científico técnico; son alumnos de intercambio académico que estudian en una universidad española durante un curso.

A continuación, detallamos las herramientas metodológicas empleadas para este estudio:

Un primer cuestionario básico aplicado en clase al principio del curso sondeó las experiencias previas de los alumnos con la traducción y, en caso afirmativo, su satisfacción al respecto.

En los primeros días de clase se realizó también una prueba escrita de redacción. Los resultados de esta prueba se compararon con la prueba final que evaluaba la producción escrita.

La traducción pedagógica se puso en práctica en el aula como actividad de consolidación de cada unidad didáctica. En esta fase del estudio se abordó el diseño y aplicación de ejercicios específicos de traducción en clase para la consolidación de conocimientos y adquisición de estrategias que resolvieran la interferencia entre el español y el inglés en parámetros cruciales en el aprendizaje/enseñanza de las dos lenguas, siguiendo a Enríquez Aranda (2003): el uso del artículo genérico y el vocabulario técnico y subtécnico de especialidad.

Para completar nuestro estudio, en la última sesión de clase un segundo cuestionario reunió las opiniones, actitudes y nivel de satisfacción del alumnado de español y de inglés como L2, (véase Apéndice I) acerca del uso de la traducción en las clases de lenguas extranjeras siguiendo en parte el modelo propuesto por Fernández-Guerra (2014).

En el examen final de la asignatura se evaluó el dominio del uso del artículo y del vocabulario específico mediante una primera prueba basada

ELIA 18, 2018, pp. 51-79～DOI: http://dx.doi.org/10.12795/elia.2018.118.03 
en traducciones de frases cortas a L2 y una segunda que consistió en un ejercicio de expresión escrita. Estas pruebas evaluaron en especial la incidencia de los parámetros tratados en la producción escrita. Finalmente, se compararon los resultados entre los dos grupos de aprendientes.

En definitiva, el estudio se ha basado en estas herramientas metodológicas y de recopilación de datos: cuestionario inicial, redacción inicial, diseño de ejercicios y práctica de traducción, cuestionario final y examen final.

Al respecto, es necesario decir que las traducciones y las redacciones se realizaron siempre en papel y sin ayuda de soporte electrónico como diccionarios.

\section{Análisis y resultados}

\subsection{Cuestionario inicial}

En el Grupo 1, los resultados del cuestionario inicial realizado al principio del curso muestran la experiencia previa de un $89 \%$ de los alumnos españoles en la práctica habitual de la traducción "silenciosa" (Hurtado Albir, 1999; Carreres, 2006), traducción de L2 a L1 como recurso para facilitar la comprensión del texto. El 83\% de los encuestados han hecho ocasionalmente ejercicios de traducción de L1 a L2 en sus estudios anteriores pero solamente para el $18 \%$ la traducción ha sido una herramienta plenamente incorporada a la metodología docente de, al menos, un curso previo. En cuanto al nivel de satisfacción con esta práctica, el $85 \%$ de los alumnos son muy escépticos ante la utilidad de la traducción para progresar en el dominio de la lengua mientras que solo el $15 \%$ tiene una actitud positiva ante la traducción como recurso docente.

En lo que respecta al grupo de estudiantes anglófonos, Grupo 2, el $75 \%$ nunca había realizado ejercicios de traducción en clase de lengua extranjera, debido, básicamente, a que tradicionalmente en EE. UU. se ha defendido el uso exclusivo de la lengua meta en el aula de lenguas por razones de índole ideológica como señala Galindo Merino (2012). A pesar de que los alumnos comentan que todos suelen traducir palabras o frases de la L2 a su L1 cuando estudian español, como señala Galindo ha sido

ELIA 18, 2018, pp. 51-79～DOI: http://dx.doi.org/10.12795/elia.2018.118.03 
habitual en las aulas norteamericanas disuadir a profesores y alumnos de una segunda lengua de utilizar la L1 en clase con el fin de no entorpecer el proceso de adquisición de la lengua.

\subsection{Prueba escrita inicial}

Tras presentar un modelo de texto argumentativo al principio de curso, los alumnos hicieron una prueba escrita donde se les pidió la redacción de un texto argumentativo sobre un tema general relacionado con la tecnología. Se trató de una prueba presencial realizada en papel en un tiempo máximo de 15 minutos y con una longitud de 150 palabras poniendo en práctica las pautas de la estructura de texto señaladas. El realizar la prueba en papel respondió a la necesidad de detectar los errores que se querían comprobar, como, por ejemplo, la ortografía, que en el caso de haberse hecho por un medio informático no se habría podido valorar de forma fiable.

Se diseñó una rúbrica para la evaluación de la prueba (véase Apéndice II). Los resultados de la prueba nos dan datos de su competencia en el uso del artículo y léxico específico, los dos temas en los que nos centramos aquí. La nota correspondiente a los dos ítems en estudio fue 5,4 puntos sobre 10 para el Grupo 1, mientras que el Grupo 2 obtuvo una media de 5,6.

Errores frecuentes detectados en el Grupo 1, en relación al uso correcto del artículo por alumnos españoles, se mostraron fundamentalmente con nombres contables plurales o nombres abstractos: *the expert systems, *the natural language processing, *the neural networks and *the robotics. Asimismo, se detectaron problemas léxicos por la elección de un vocablo incorrecto como en el caso de la traducción de campo por camp, en lugar de field, entre otras. Las faltas de ortografía en términos técnicos fueron frecuentes.

En cuanto al Grupo 2, alumnos angloparlantes, la traducción al español se compone de nombres abstractos como "la informática" y sustantivos plurales como "los ordenadores" y se observó que se repetían frases del tipo *ordenadores han cambiado el mundo, en las que el estudiante omite el artículo determinado en su valor de referencia genérica 
en español. En cuanto al léxico, fueron frecuentes los errores de género en determinados sustantivos como, por ejemplo, *la sistema, y los errores ortográficos de acentuación y de uso de consonantes dobles.

\subsection{Diseño de ejercicios y práctica de la traducción pedagógica}

Se diseñaron frases cortas para traducir en la lengua meta siguiendo a Alcarazo López y López Fernández (2014). Estas frases, de acuerdo con Cook (2010) y García Castañón (2014), tienen como objetivo la puesta en práctica de los contenidos tratados en cada unidad para reforzar los conocimientos del estudiante.

A título de ejemplo, la gramática de una unidad del temario del curso se centró en la referencia genérica en inglés y en español, un tema de especial dificultad para aprendientes de ambas lenguas, a pesar de que autores como Navarro (2008) señalan que, en su caso, en el lenguaje científico y el de la prensa en español, la omisión del artículo a la manera de la lengua inglesa es una práctica común hoy en día como parte de lo que denomina anglización del español.

A continuación, ofrecemos una muestra de errores comunes acerca de la interferencia de la L1 detectados en el aula.

\subsubsection{Interferencias sintácticas: uso del artículo de referencia genérica}

Las traducciones que detallamos a continuación evidencian que alumnos de inglés (L2), nivel B2, no dominan las reglas del artículo en inglés, concretamente la ausencia del artículo para expresar referencia genérica en los plurales y los nombres abstractos. Por otra parte, los aprendientes anglófonos en la traducción al español no hacen uso del artículo y aplican las normas propias de su lengua al respecto.

En las siguientes tablas, cada columna L1 y L2 incluye ejemplos que se corresponden con los dos grupos diferentes de estudiantes: alumnos hispanófonos (Grupo 1) y alumnos anglófonos (Grupo 2).

ELIA 18, 2018, pp. 51-79 DOI: http://dx.doi.org/10.12795/elia.2018.i18.03 


\begin{tabular}{|l|l|}
\hline \multicolumn{1}{|c|}{ Frase en L1 } & \multicolumn{1}{c|}{ Traducción a L2 } \\
\hline $\begin{array}{l}\text { GRUPO 1. La inteligencia artificial es } \\
\text { la rama de la ciencia informática cuyo } \\
\text { objetivo es hacer que los ordenadores } \\
\text { se comporten como humanos. }\end{array}$ & $\begin{array}{l}\text { *The artificial intelligence is the branch } \\
\text { of computer science whose objective is } \\
\text { to make *the computers behave like } \\
\text { humans. (omisión del artículo) }\end{array}$ \\
\hline $\begin{array}{l}\text { GRUPO 2. Many techniques have been } \\
\text { developed to improve data security. }\end{array}$ & $\begin{array}{l}\text { Se han desarrollado muchas técnicas para } \\
\text { mejorar *seguridad de *datos. (la, los) }\end{array}$ \\
\hline
\end{tabular}

Tabla 1. Ejemplos de frases con uso incorrecto del artículo en L2 de aprendientes españoles y anglófonos.

En el primer grupo, la traducción al inglés presenta varios puntos críticos como son artificial intelligence, nombre abstracto sin artículo, así como computers, contable plural. En el segundo, la traducción del inglés ha omitido los artículos determinados en español de la seguridad de los datos, lo que Miletich (2008) denomina anglicismos sintácticos.

\subsubsection{Interferencias léxicas}

a) Utilización de vocablos inexistentes en L2 pero con forma semejante a la Ll.

\begin{tabular}{|c|c|}
\hline Frase en L1 & Traducción a L2 \\
\hline $\begin{array}{l}\text { GRUPO 1. Las redes neuronales } \\
\text { procesan la información como } \\
\text { respuesta a inputs externos. } \\
\text { Los sistemas operativos multiusuario } \\
\text { permiten que múltiples usuarios } \\
\text { puedan acceder a un único sistema. }\end{array}$ & $\begin{array}{l}\text { *Neuronal networks process } \\
\text { information as a response to *extern } \\
\text { inputs. (neural) (external) } \\
\text { A multiuser *operative system allows } \\
\text { multiple users to *acceed an only } \\
\text { system. (operating, access) }\end{array}$ \\
\hline $\begin{array}{l}\text { GRUPO 2. Computing has changed } \\
\text { our world. } \\
\text { In conventional computer systems, } \\
\text { the computer hardware does not } \\
\text { execute the user's source program. }\end{array}$ & $\begin{array}{l}\text { La *computando ha cambiado nuestro } \\
\text { mundo. (informática) } \\
\text { En los sistemas informáticos } \\
\text { *convenciales, el hardware } \\
\text { informático no ejecuta el programa } \\
\text { fuente del usuario. (convencionales) }\end{array}$ \\
\hline
\end{tabular}

Tabla 2. Ejemplos de frases con vocablos inexistentes en L2 de aprendientes españoles y anglófonos.

ELIA 18, 2018, pp. 51-79 DOI: http://dx.doi.org/10.12795/elia.2018.i18.03 
Otros términos frecuentes en el campo de las tecnologías de la información que fueron objeto de malas traducciones son, por ejemplo, *procesament (processing) y *dispositives (devices). En el Grupo 2 son igualmente frecuentes errores como *usario (usuario) o *desarollar (desarrollar).

b. Utilización de palabras en L2 a las que, por su semejanza con la L1, se les atribuye erróneamente el mismo significado que en L1: falsos amigos o falsos cognados. Se trata de un error de significado al usar una palabra real en español/inglés con un significado diferente.

\begin{tabular}{|l|l|}
\hline \multicolumn{1}{|c|}{ Frase en L1 } & \multicolumn{1}{|c|}{ Traducción a L2 } \\
\hline $\begin{array}{l}\text { GRUPO 1. Las tecnologías actuales } \\
\text { permiten una mayor eficiencia y un } \\
\text { mayor nivel de calidad. }\end{array}$ & $\begin{array}{l}\text { *Actual technologies allow greater } \\
\text { efficiency and greater quality level. } \\
\text { (current) }\end{array}$ \\
\hline $\begin{array}{l}\text { GRUPO 2. Current computers are } \\
\text { much faster than the computers that } \\
\text { were used ten years ago. }\end{array}$ & $\begin{array}{l}\text { Los ordenadores *corrientes son más } \\
\text { rápidos que los ordenadores que } \\
\text { usaban hace diez años (actuales). }\end{array}$ \\
\hline
\end{tabular}

Tabla 3. Ejemplos de frases con falsos amigos de aprendientes españoles y anglófonos.

Un $20 \%$ de los alumnos españoles mostró interferencias con su L1 al traducir frases que contenían vocablos técnicos y subtécnicos habituales de su campo como, por ejemplo: *dates (por datos o información) en lugar de data, *exit (por éxito) en lugar de success, *target (por tarjeta) en lugar de card, o *assist (por asistir) en lugar de attend.

En cuanto a los alumnos de habla inglesa, Grupo 2, un $25 \%$ mostró interferencias con su lengua materna en la traducción de léxico como *soportar (por support) en lugar de apoyar, *realizar (por realize) en lugar de darse cuenta, *atender (por attend) en lugar de asistir, *recordar (por record) en lugar de grabar, *corriente (por current) en lugar de actual o *tópico (por topic) en lugar de tema.

c. La correcta ortografía del vocabulario específico es otro de los aspectos que se persigue afianzar mediante la traducción, sobre todo en

ELIA 18, 2018, pp. 51-79 DOI: http://dx.doi.org/10.12795/elia.2018.i18.03 
palabras que son muy similares en L1 y L2 y son objeto de frecuentes interferencias.

\begin{tabular}{|l|l|}
\hline \multicolumn{1}{|c|}{ Frase en L1 } & \multicolumn{1}{c|}{ Traducción a L2 } \\
\hline $\begin{array}{l}\text { GRUPO 1. inteligencia artificial } \\
\text { lenguajes de programación }\end{array}$ & $\begin{array}{l}\text { artificial *inteligence (intelligence) } \\
\text { *programing lenguajes } \\
\text { (programming languages) }\end{array}$ \\
\hline $\begin{array}{l}\text { GRUPO 2. data compressing program } \\
\text { Most current computers are sequential }\end{array}$ & $\begin{array}{l}\text { *compressor de datos (compresor) } \\
\text { La mayoría de computadoras actuales } \\
\text { son *sequenciales (secuenciales) }\end{array}$ \\
\hline
\end{tabular}

Tabla 4. Ejemplos de vocabulario con errores ortográficos en L2 de aprendientes españoles y anglófonos.

En cuanto a los aprendientes anglófonos, sus errores ortográficos más comunes tienen que ver tanto con la no aplicación de las reglas de acentuación como con las restricciones propias del español en lo que atañe a la posibilidad de combinar determinadas consonantes o doblarlas.

d. En cuanto al género de las palabras en español, los alumnos del Grupo 2 han estudiado en niveles anteriores que los sustantivos que acaban en $-\mathrm{o}$ son masculinos y los que terminan en -a femeninos y aplican este conocimiento sin tener en cuenta las múltiples excepciones; de ese modo, la hipergeneralización de esta regla favorece los errores de género en cierto tipo de palabras que por su etimología no la cumplen, errores que se hacen patentes tanto en los artículos determinados como en los adjetivos que acompañan a estos sustantivos.

\subsection{Cuestionario final}

Una posterior fase del estudio consistió en el diseño y cumplimentación de una encuesta para valorar la motivación y grado de satisfacción del alumnado con las actividades de traducción desarrolladas. Este cuestionario final (Apéndice I) revela una actitud más favorable hacia la traducción que la detectada en el cuestionario anterior. El $90 \%$ de los encuestados españoles está de acuerdo, o muy de acuerdo, en que la traducción L1-L2 debe formar parte de un curso de lengua extranjera porque es un reto que

ELIA 18, 2018, pp. 51-79 DOI: http://dx.doi.org/10.12795/elia.2018.i18.03 
ayuda a mejorar la gramática y el vocabulario de la L2. Los alumnos sitúan la traducción muy por encima de otras actividades del curso centradas en la comprensión lectora y comprensión y expresión oral. En su opinión, la traducción contribuye a reforzar las estructuras gramaticales $(54 \%)$ y vocabulario (46\%). Es interesante señalar que hubo sugerencias en favor de la traducción directa en los comentarios adicionales, por ejemplo: "sería también buena idea incluir ejercicios desde la L2 a la L1".

De igual modo, en los aprendientes anglófonos los errores ortográficos más repetidos anteriormente mejoraron como las consonantes dobles en *efficiente, *intelligencia, *programmación, *compressor, o las tildes en términos como *movil, *tecnica, *informatica. Todos los encuestados anglófonos están totalmente de acuerdo o de acuerdo en que su conocimiento de vocabulario específico mejora cuando practica la traducción en clase de LFE.

Su visión de la traducción como herramienta para la mejora de sus habilidades lingüísticas en clase de LFE es claramente positiva: la totalidad del alumnado está completamente de acuerdo o de acuerdo en que los ejercicios de traducción son entretenidos; en que les ayudan a darse cuenta de las diferencias entre los sistemas lingüísticos españoles e ingleses; y en que ayudan al aprendizaje de la L2, en línea con los resultados al respecto de Fernández-Guerra (2014). Asimismo, todos son partidarios de la incorporación de ejercicios de traducción en el aula.

Cabe destacar que, por una parte, la traducción como ejercicio para practicar la gramática la considera muy importante la mitad de los estudiantes, muy por encima de otras destrezas tales como la comprensión escrita, la expresión escrita o la expresión oral. Por otra, también se valora muy positivamente como recurso pedagógico para practicar el vocabulario ocupando el primer lugar para un $25 \%$ del alumnado; si bien queda en segundo lugar de preferencia, sobrepasada por la comprensión oral.

Un $73 \%$ de los alumnos españoles defiende la tesis de que la traducción es una destreza que debe incorporarse a los contenidos de los cursos de lengua.

Los comentarios que realizan libremente sobre este tipo de ejercicios son positivos, por ejemplo, en los anglófonos "me gusta el ejercicio de la 
traducción porque puedo utilizar mi lengua materna para aprender más en mi lengua secundaria", "los ejercicios de traducción especialmente me ayudan a aprender palabras y practicar la gramática y el orden de las palabras", "me ayuda a entender"...

\subsection{Prueba final}

Como parte del examen final, se incluyeron frases de traducción y también un ejercicio de expresión escrita de 150 palabras aproximadamente sobre este tema: la globalización y las nuevas tecnologías. Estas dos pruebas iban orientadas a comprobar la competencia del alumno en el uso del artículo y vocabulario técnico y subtécnico. Para ello se utilizó la misma rúbrica que se diseñó para la prueba inicial.

Los resultados del Grupo 1 mostraron una variación absoluta en los parámetros de estudio de 1,4 mientras que el Grupo 2 obtuvo una media de variación absoluta de 1,8:

\begin{tabular}{|c|c|c|c|}
\cline { 2 - 4 } \multicolumn{1}{c|}{} & Prueba inicial & Prueba final & Variación \\
\hline Grupo 1 & 5,4 & 6,8 & 1,4 \\
\hline Grupo 2 & 5,6 & 7,4 & 1,8 \\
\hline
\end{tabular}

Tabla 5: Comparación de resultados de la prueba inicial y la prueba final

En las dos pruebas, los alumnos de ambos grupos fueron más conscientes del uso y omisión del artículo para expresar referencia genérica o específica, nombres contables e incontables. Pese a todo, todavía se observan problemas en su utilización, incluso en alumnos que destacan por una mayor competencia en el uso de la lengua.

En cuanto al léxico en alumnos del Grupo 1, la reiterada traducción de frases que contenían términos como sistemas, caracteres, conexión, física, ingeniero, silicio, grafito, técnico contribuyó a afianzar su correcta ortografía en inglés: systems, character, connection, engineer, silicon, graphite, technician, frente a los errores habituales *sistems, ${ }^{*}$ caracter, *conextion, *phisics, *ingeniers, *silice, *grafit, *technique. Es interesante 
observar la incidencia de algún error que persistió en alguna palabra como es el caso de technique (*thechnique, *technike) pese a su práctica repetida en clase.

Del mismo modo, en lo que respecta al Grupo 2, la frecuencia en la aparición de términos como, por ejemplo, sistema, programa, tema, generación, ingeniero, física en sus traducciones fue determinante tanto para la interiorización del género correcto de estas palabras como para su correcta ortografía, evitando finalmente errores como *la sistema, *la programa, *la tema, *el generacion, *engeniero o *fisical.

La mejora de los resultados obtenidos en la prueba de producción escrita final con respecto a los detectados en la prueba inicial corrobora que la traducción ha contribuido a evitar la interferencia con la L1, redundando así en una mejora de la expresión escrita.

\section{Conclusión}

Con la incorporación de la traducción al aula estamos asistiendo al paso de un antiguo mito a una realidad. Con ello, los antiguos modelos en los que la traducción ha tenido un papel relevante están detrás de los nuevos enfoques y metodologías (Argüelles Álvarez, 2006). Los resultados positivos de la realización de ejercicios prácticos de traducción referidos en este trabajo están en consonancia con los obtenidos por Calis y Dikilitas (2012) y Fernández-Guerra (2014).

Con respecto a la primera hipótesis planteada, nuestros resultados han mostrado una menor interferencia con la L1 con el uso pedagógico de la traducción, confirmando la mejora del dominio del artículo de referencia genérica en el aula de LFE y en el dominio de vocabulario de especialidad. Las frases cortas diseñadas para practicar dichas estructuras gramaticales y vocabulario específico de tecnologías de la información resaltan las diferencias entre los dos sistemas lingǘsticos y evidencian los problemas reales de aprendizaje.

Nuestro trabajo confirma parcialmente la segunda hipótesis planteada, la similitud de las interferencias en la producción escrita en función de la lengua materna de los dos grupos, al detectar los mismos

ELIA 18, 2018, pp. 51-79～DOI: http://dx.doi.org/10.12795/elia.2018.118.03 
problemas en el uso del artículo en ambos. Se observan, no obstante, diferencias en cuanto al léxico. Un hecho evidente revelado en el Grupo 1 es que, si bien por lo general la frecuente etimología latina del vocabulario de especialidad facilita su comprensión al alumno español, en el caso que nos ocupa, esta similitud con el español ha dificultado su correcta traducción, al manifestar el alumno una clara tendencia a reflejar la forma ortográfica original del español en la L2. Por su parte, los alumnos anglófonos del Grupo 2 presentan dos problemas adicionales con respecto al Grupo 1. En primer lugar, la dificultad para identificar e interiorizar el género de determinadas palabras a las que, normalmente por su terminación, les atribuyen un género erróneo. En segundo lugar, la dificultad en la correcta utilización del acento ortográfico.

La mejora de la expresión escrita tras la práctica de la traducción pedagógica planteada en nuestra tercera hipótesis, ha sido sustancial en la prueba final, evidenciando un mayor conocimiento y aplicación efectiva de las reglas de funcionamiento del sistema de la lengua y de las estrategias necesarias para interactuar lingüísticamente de una manera adecuada. Los reiterados ejercicios de traducción les han servido para consolidar la correcta forma de los términos y evitar formas inexistentes, falsos amigos o faltas ortográficas en L2.

Este estudio ha señalado una mayor progresión de los alumnos angloparlantes en el dominio de la comunicación escrita con respecto a los españoles. Al igual que en el caso de Fernández-Guerra (2014), más contacto con la L2 por vivir en España durante una estancia prolongada ha podido contribuir a una mejora destacada en el aprendizaje de esta destreza.

En cuanto a la satisfacción del alumno con respecto a la práctica de la traducción, objeto de nuestra última hipótesis, los alumnos han manifestado en el cuestionario final más motivación y una actitud más favorable hacia la traducción para consolidar estructuras practicadas en el aula. Los alumnos ven esta práctica como una estrategia que les ayuda a comparar y contrastar la lengua propia y la lengua meta y a descubrir diferencias que antes habían pasado por alto. A la vez que una mayor motivación e implicación de los alumnos en el proceso de aprendizaje, la traducción les ayuda a sistematizar y racionalizar su aprendizaje (Carreres, 2006). 
El trabajo que presentamos aquí es una primera aproximación al papel de la traducción en la clase de lenguas de especialidad. El análisis de otros problemas de aprendizaje del español e inglés como, por ejemplo, el orden de las palabras, puede seguir esta línea investigadora que intenta, sin lugar a dudas, resaltar la importancia de este recurso de aprendizaje y darle su merecido espacio en nuestras aulas.

\section{Referencias bibliográficas}

Alcarazo López, N. y López Fernández, N. (2014). Aplicaciones prácticas de la traducción pedagógica en la clase de ELE [On-line]. RedELE, 26. https:// www.mecd.gob.e s/da m/j cr:35562027-c807-4b 7 c-b91669e3956baac9/2014-redele-26-00alcarazo-lopez-pdf.pdf

Alexopoulou, A. (2010). El papel de la transferencia en los errores léxicos [Online]. Revista Nebrija de Lingüistica Aplicada, 5(3). https://www.nebrija. com/revista-linguistica/files/articulosPDF/articulo_530b40943d088.pdf

Argüelles Álvarez, I. (2006). Comprensión lectora en IFE: las viejas propuestas en nuevos contextos [On-line]. Revista de Lenguas para Fines Especificos, 11-12, 13-32. https://dialnet.unirioja.es/descarga/articulo/2116384.pdf

Caballero Rodríguez, B. (2010). El papel de la traducción en la enseñanza del español. En Instituto Cervantes (Ed.), Selección de artículos del I Congreso de Español como lengua Extranjera en Asia-Pacífico (I CELEAP, Manila 2009). El Currículo de E/LE en Asia-Pacifico (pp. 339-352). Centro Virtual Cervantes. https://cvc.cervantes.es/ensenanza/biblioteca_ele/ publicaciones_centros/pdf/manila_2009/22_aplicaciones_09.pdf

Calis, E., y Dikilitas, K. (2012). The use of translation in EFL classes as L2 learning practice. Procedia-Social and Behavioral Sciences, 46, 50795084 .

Carreres, A. (2006). Strange bedfellows: Translation and Language teaching. The teaching of translation in Modern Languages Degrees: Uses and Limitations [On-line]. En Sixth Symposium on Translation, Terminology and Interpretation in Cuba and Canada, December 2006. Canadian Translators, Terminologists and Interpreters Council. http://www.cttic.org/ACTI/2006/ papers/Carreres.pdf

ELIA 18, 2018, pp. 51-79

DOI: http://dx.doi.org/10.12795/elia.2018.i18.03 
Carreres, A., Muñoz-Calvo, M. y M. Noriega-Sánchez (2017). Translation in Spanish language teaching: the fifth skill/La traducción en la enseñanza del español: la quinta destreza. Journal of Spanish Language Teaching, 4(2), 99-109.

Consejo de Europa. (2002). Marco Común Europeo de Referencia para las Lenguas: aprendizaje, enseñanza, evaluación. Madrid: MECD-Anaya. https://cvc.cervantes.es/ensenanza/biblioteca_ele/marco/cvc_mer.pdf

Cook, G. (2010). Translation in Language Teaching:AnArgument for Reassessment. Oxford: Oxford University Press.

Duff, A. (1989). Translation. Oxford: Oxford University Press.

Ellis, R. (1994). The Study of Second Language Acquisition. Oxford: Oxford University Press.

Enríquez Aranda, M. (2010). La traducción en la enseñanza y en el aprendizaje de la lengua inglesa: materiales de estudio [On-line]. Elia, 4, 117-137. http:// institucional.us.es/revistas/elia/4/7.\%20enriquez.pdf

Fernández-Guerra, A. B. (2014). The Usefulness of Translation in Foreign Language Learning: Students' Attitudes. International Journal of English Language \& Translation Studies, 2(1), 153-170.

Galindo Merino, M. M. (2012). La lengua materna en el aula de ELE [On-line]. Málaga: ASELE. www.cervantesvirtual.com/descargaPdf/la-lenguamaterna-en-el-aula-de-ele

García Castañón, J. M. (2014). La traducción pedagógica en la enseñanza de español como lengua extranjera. (Tesis de Máster). Universidad de Oviedo.

Gile, D. (1995). Basic Concepts and Models for Interpreter and Translator Training. Amsterdam y Filadelphia: John Benjamins.

Hurtado Albir, A. (1999). Enseñar a traducir. Madrid: Edelsa.

Instituto Cervantes. (2006). Plan Curricular del Instituto Cervantes. Niveles de referencia para el español. B1-B2 Madrid: Instituto Cervantes-Biblioteca Nueva.

Lado, R. (1957). Linguistics Across Cultures: Applied Linguistics for Language Teachers. Ann Arbor, MI: University of Michigan Press.

ELIA 18, 2018, pp. 51-79 DOI: http://dx.doi.org/10.12795/elia.2018.i18.03 
Malmkjaer, K. (Ed) (1998). Translation and Language Teaching. Londres y Nueva York: Routledge.

Miletich, M. (2008). Pedagogía del error: aspectos didácticos de la traducción. En L. González y P. Hernúñez (Eds.), Actas del III Congreso Internacional: el Español, Lengua de Traducción, 2006 Puebla (México). Traducción: Contacto y Contagio (pp. 453-465). Bruselas: Esletra.

Navarro, F. (2008). La anglización del español: mucho más allá de bypass, piercing, test, airbag, container y spa. En L. González y P. Hernúñez (Eds.), Actas del III Congreso Internacional: el Español, Lengua de Traducción, 2006 Puebla (México). Traducción: Contacto y Contagio (pp. 213-232). Bruselas: Esletra.

Pérez-Sabater, C. y B. Montero-Fleta, B. (2015). ESP vocabulary and social networking: The case of Twitter. Ibérica, 29, 129-154.

Popovic, R, (2001). The Place of Translation in Language Teaching, Bridges. The Journal of the Thrace-Macedonia Teachers'Association, 5, 3-8.

Quirk, R. y S. Greenbaum (2002). A University Grammar of the English Language. Londres y Nueva York: Longman.

Snape, N., García-Mayo, M. D., y A. Gürel (2013). L1 transfer in article selection for generic reference by Spanish, Turkish and Japanese L2 learners. International Journal of English Studies, 13(1), 1-28.

Strevens, P. (1977). New Orientations in the Teaching of English. Nueva York: Oxford University Press.

Vermes, A. (2010). Translation in foreign language teaching: a brief overview of pros and cons. Eger Journal of English Studies, 10, 83-93.

Zanettin, F. (2009). Corpus-based translation activities for language learners. The Interpreter and Translator Trainer, 3(2), 209-224.

ELIA 18, 2018, pp. 51-79 DOI: http://dx.doi.org/10.12795/elia.2018.i18.03 


\section{Apéndices}

\section{Apéndice I}

Rúbrica para evaluación inicial y final b2

\begin{tabular}{|l|l|l|l|l|}
\hline \multicolumn{1}{|c|}{ Tipo de errores } & $\mathbf{9 - 1 0}$ & $\mathbf{7 - 8}$ & $\mathbf{5 - 6}$ & $\mathbf{0 - 5}$ \\
\hline \multicolumn{1}{|c|}{ Interferencias sintácticas } & & & & \\
\hline $\begin{array}{l}\text { Uso artículo referencia genérica en nombre } \\
\text { contable singular }\end{array}$ & & & & \\
\hline $\begin{array}{l}\text { Uso artículo referencia genérica nombre contable } \\
\text { plural }\end{array}$ & & & & \\
\hline $\begin{array}{l}\text { Uso artículo referencia genérica en nombre } \\
\text { abstracto }\end{array}$ & & & & \\
\hline $\begin{array}{l}\text { Uso artículo en nombre seguido de } \\
\text { postmodificación }\end{array}$ & & & & \\
\hline Utilización de vocablos inexistentes & & & & \\
\hline Utilización de falsos amigos & & & & \\
\hline Errores ortográficos & & & & \\
\hline Errores de acentuación & & & & \\
\hline
\end{tabular}

\begin{tabular}{|c|l|l|}
\hline Puntuación otorgada & & \\
\hline $9-10:$ & Muy competente & no tiene errores \\
\hline $7-8:$ & Competente & tiene pocos errores \\
\hline $5-6$ & Aceptable & tiene errores \\
\hline $0-5$ & No competente & tiene muchos errores \\
\hline
\end{tabular}




\section{Apéndice II}

\section{Cuestionario para alumnos de LSP}

\begin{tabular}{|l|l|}
\hline ¿Es esta la primera vez que haces ejercicios de traducción en clase? \\
\hline Sí & \\
\hline No & \\
\hline
\end{tabular}

\begin{tabular}{|l|l|}
\hline Suelo traducir de español a inglés cuando estudio español \\
\hline Totalmente de acuerdo & \\
\hline De acuerdo & \\
\hline Ni de acuerdo ni en desacuerdo & \\
\hline En desacuerdo & \\
\hline Totalmente en desacuerdo & \\
\hline
\end{tabular}

\begin{tabular}{|l|l|}
\hline Normalmente traduzco de español a inglés cuando estudio español \\
\hline Solo palabras & \\
\hline Frases completas & \\
\hline Párrafos completos & \\
\hline
\end{tabular}

\begin{tabular}{|l|l|}
\hline $\begin{array}{l}\text { Mi dominio de la gramática española mejora cuando practico la traducción } \\
\text { en clases de LSP }\end{array}$ \\
\hline Totalmente de acuerdo & \\
\hline De acuerdo & \\
\hline Ni de acuerdo ni en desacuerdo & \\
\hline En desacuerdo & \\
\hline Totalmente en desacuerdo & \\
\hline
\end{tabular}

ELIA 18, 2018, pp. 51-79 DOI: http://dx.doi.org/10.12795/elia.2018.i18.03 


\begin{tabular}{|l|l|}
\hline \multicolumn{2}{|l|}{$\begin{array}{l}\text { El dominio del vocabulario especifico de comunicación mejora cuando } \\
\text { practico la traducción en clases de LSP }\end{array}$} \\
\hline Totalmente de acuerdo & \\
\hline De acuerdo & \\
\hline Ni de acuerdo ni en desacuerdo & \\
\hline En desacuerdo & \\
\hline Totalmente en desacuerdo & \\
\hline
\end{tabular}

\begin{tabular}{|l|l|}
\hline $\begin{array}{l}\text { Mi conocimiento del orden de las palabras en español mejora cuando } \\
\text { practico la traducción en clases de LSP }\end{array}$ \\
\hline Totalmente de acuerdo & \\
\hline De acuerdo & \\
\hline Ni de acuerdo ni en desacuerdo & \\
\hline En desacuerdo & \\
\hline Totalmente en desacuerdo & \\
\hline
\end{tabular}

\begin{tabular}{|l|l|}
\hline \multicolumn{2}{|l|}{ Los ejercicios de traducción suponen para mí un reto } \\
\hline Totalmente de acuerdo & \\
\hline De acuerdo & \\
\hline Ni de acuerdo ni en desacuerdo & \\
\hline En desacuerdo & \\
\hline Totalmente en desacuerdo & \\
\hline
\end{tabular}

ELIA 18, 2018, pp. 51-79～DOI: http://dx.doi.org/10.12795/elia.2018.i18.03 
77 Inmaculada Barbasán Ortuño, Carmen Pérez-Sabater, Begoña Montero-Fleta

\begin{tabular}{|l|l|}
\hline \multicolumn{2}{|l|}{ Los ejercicios de traducción me resultan entretenidos } \\
\hline Totalmente de acuerdo & \\
\hline De acuerdo & \\
\hline Ni de acuerdo ni en desacuerdo & \\
\hline En desacuerdo & \\
\hline Totalmente en desacuerdo & \\
\hline
\end{tabular}

\begin{tabular}{|l|l|}
\hline $\begin{array}{l}\text { Los ejercicios de traducción me ayudan a darme cuenta de las diferencias } \\
\text { entre los sistemas lingüisticos españoles e ingleses }\end{array}$ \\
\hline Totalmente de acuerdo & \\
\hline De acuerdo & \\
\hline Ni de acuerdo ni en desacuerdo & \\
\hline En desacuerdo & \\
\hline Totalmente en desacuerdo & \\
\hline
\end{tabular}

\begin{tabular}{|l|l|}
\hline Los ejercicios de traducción ayudan al aprendizaje de la lengua extranjera \\
\hline Totalmente de acuerdo & \\
\hline De acuerdo & \\
\hline Ni de acuerdo ni en desacuerdo & \\
\hline En desacuerdo & \\
\hline Totalmente en desacuerdo & \\
\hline
\end{tabular}

ELIA 18, 2018, pp. 51-79 DOI: http://dx.doi.org/10.12795/elia.2018.i18.03 


\begin{tabular}{|l|l|}
\hline Los cursos de lenguas deberían incorporar el ejercicio de la traducción \\
\hline Totalmente de acuerdo & \\
\hline De acuerdo & \\
\hline Ni de acuerdo ni en desacuerdo & \\
\hline En desacuerdo & \\
\hline Totalmente en desacuerdo & \\
\hline
\end{tabular}

\begin{tabular}{|l|l|}
\hline $\begin{array}{l}\text { No soy partidario/a de la traducción porque el uso de la lengua materna en } \\
\text { clase de LSP influye de forma negativa en el aprendizaje de la lengua }\end{array}$ \\
\hline Totalmente de acuerdo & \\
\hline De acuerdo & \\
\hline Ni de acuerdo ni en desacuerdo & \\
\hline En desacuerdo & \\
\hline Totalmente en desacuerdo & \\
\hline
\end{tabular}

\begin{tabular}{|l|l|}
\hline Para practicar la gramática mi destreza favorita es \\
\hline Comprensión oral & \\
\hline Comprensión lectora & \\
\hline Expresión escrita & \\
\hline Traducción & \\
\hline Ejercicios de práctica de la gramática & \\
\hline Expresión oral & \\
\hline
\end{tabular}

ELIA 18, 2018, pp. 51-79 DOI: http://dx.doi.org/10.12795/elia.2018.i18.03 
79 Inmaculada Barbasán Ortuño, Carmen Pérez-Sabater, Begoña Montero-Fleta

\begin{tabular}{|l|l|}
\hline \multicolumn{2}{|l|}{ Para practicar el vocabulario español mi destreza favorita es } \\
\hline Comprensión oral & \\
\hline Comprensión lectora & \\
\hline Expresión escrita & \\
\hline Traducción & \\
\hline Ejercicios de práctica de la gramática & \\
\hline Expresión oral & \\
\hline
\end{tabular}

Comentarios acerca de lo que me gusta y lo que no me gusta de usar la traducción en clase

First version received: July, 2018

Final version accepted: October, 2018

ELIA 18, 2018, pp. 51-79ＯDOI: http://dx.doi.org/10.12795/elia.2018.i18.03 